Mathematical Modelling and Analysis

Volume 20 Number 3, May 2015, 396-408

http://dx.doi.org/10.3846/13926292.2015.1049571

(C) Vilnius Gediminas Technical University, 2015
Publisher: Taylor\&Francis and VGTU

http://www.tandfonline.com/TMMA

ISSN: $1392-6292$

eISSN: $1648-3510$

\title{
A Wavelet Based Alternative Iteration Method for the Orientation Refinement of Cryo-Electron Microscopy 3D Reconstruction
}

\section{Zhucui Jing ${ }^{a}$ and Ming $\mathbf{L i}^{b}$}

\author{
${ }^{a}$ School of Economics and Management, Beijing Jiaotong University \\ 100044 Beijing, China \\ ${ }^{b}$ LSEC, ICMSEC, Academy of Mathematics and Systems Science, Chinese \\ Academy of Sciences \\ 100190 Beijing, China
}

E-mail: liming@lsec.cc.ac.cn

Received March 25, 2014; revised March 6, 2015; published online May 15, 2015

\begin{abstract}
Cryo-electron microscopy (cryo-EM) single particle method (SPM) reconstructs the three-dimensional (3D) density map of biological macromolecules using $2 \mathrm{D}$ particle images with estimated orientations. The estimated orientations have errors which result in the decrease in resolution of the reconstructed map. We propose a wavelet orthonormal bases based iteration method by refining alternatively the orientations and the map using Levenberg-Marquardt algorithm and soft-thresholding, respectively. The convergence analysis of the proposed algorithm is provided and numerical experiments for simulated particle images show its good performance.
\end{abstract}

Keywords: alternating-direction method, convergence analysis, inverse problem, nonlinear least squares, Haar wavelet.

AMS Subject Classification: 68U10; 65J22; 65T60; 94A08; 90C90.

\section{Introduction}

In the past few decades, with the advance in the electron microscopes, specimen preparation methods and image processing techniques, cryo-EM techniques $[6,8,12,13,17,24]$ have become indispensable tools for determining the $3 \mathrm{D}$ structures of biological macromolecules, macromolecular complexes and cells. Among all computerized techniques used in the cryo-EM technique, the SPM is one of the most popular ones. The image processing in the SPM includes image alignment and classification; image filtering and contrast transfer function correction; and 3D reconstruction (see $[13,16,17,20,24]$ for more details). These modules are integrated in an iterative framework to iteratively refine the 3D map using the projection matching method [23]. 
The purpose of this paper is to develop a new algorithm to refine the reconstructed maps of cryo-EM SPM so as to obtain better resolutions. The basis principle of cryo-EM SPM 3D reconstruction lies in the 3D X-ray transform. Let $\mathbf{x}-\mathbf{y}-\mathbf{z}$ be the global coordinate system and $(\theta, \phi, \psi)$ be three Euler angles, then the rotation matrix according to the Euler angles using $\mathbf{z}-\mathbf{y}-\mathbf{z}$ convention is defined as

$$
\begin{aligned}
& R=\left(\mathbf{e}_{1}, \mathbf{e}_{2}, \mathbf{d}\right)^{\top}:= \\
& \left(\begin{array}{ccc}
\cos \theta \cos \phi \cos \psi-\sin \theta \sin \psi & \sin \theta \cos \phi \cos \psi+\cos \theta \sin \psi & -\sin \phi \cos \psi \\
-\cos \theta \cos \phi \sin \psi-\sin \theta \cos \psi & -\sin \theta \cos \phi \sin \psi+\cos \theta \cos \psi & \sin \phi \sin \psi \\
\cos \theta \sin \phi & \sin \theta \sin \phi & \cos \phi
\end{array}\right),
\end{aligned}
$$

where $\mathbf{e}_{\mathbf{1}}-\mathbf{e}_{\mathbf{2}}-\mathbf{d}$ defines the new local coordinate system after rotation, and $\mathbf{d}=(\cos \theta \sin \phi, \sin \theta \sin \phi, \cos \phi)^{\top} \in \mathbb{S}^{2}$ is the projection direction vector on the unit sphere $\mathbb{S}^{2}$. The $\mathbf{z}-\mathbf{y}-\mathbf{z}$ convention defines the rotation matrix $R$ by first rotating around the original $z$ axis, then rotating around the new $y$ axis, and finally rotating around the new $z$ axis. The $3 \mathrm{D}$ X-ray transform (projection) $\mathcal{P}$ and back-projection $\mathcal{P}^{*}$ of function $f: \mathbb{R}^{3} \mapsto \mathbb{R}$ along the direction $\mathbf{d}$ are defined as

$$
g(\mathbf{d}, x, y)=\mathcal{P}(\theta, \phi, \psi, x, y):=\int_{\mathbb{R}} f\left(x \mathbf{e}_{1}+y \mathbf{e}_{2}+t \mathbf{d}\right) d t
$$

and

$$
\left(\mathcal{P}^{*} g\right)(\mathbf{x}):=\int_{\mathbb{S}^{2}} g\left(\mathbf{d},\left\langle\mathbf{x}, \mathbf{e}_{1}\right\rangle,\left\langle\mathbf{x}, \mathbf{e}_{2}\right\rangle\right) d \mathbf{d}
$$

respectively.

For every orientation $(\theta, \phi, \psi)$, a $2 \mathrm{D}$ particle image $g$, is imaged using transmission electron microscope, which can be described as a group of 3D $\mathrm{X}$-ray transform for that orientation $(\theta, \phi, \psi)$. The cryo-EM SPM imaging can produce many particles images, each being a $2 \mathrm{D}$ projection of one biological macromolecule with a randomly produced orientation $(\theta, \phi, \psi)$. For SPM, all the macromolecules are identical, hence we can obtain many projections of the macromolecule with different orientations. The orientations can then be estimated using common-line based methods $[5,6,14,16]$ and thereafter be used to reconstruct an initial map. Since these particle images are picked up from the original micrographs, the centers of the particles usually deviate from the images' centers. Therefore, translational alignments among these particles should be performed before 3D reconstruction. Totally, we need five parameters $\left(\theta, \phi, \psi, \delta_{x}, \delta_{y}\right)$ in order to do reconstruction. As a result, we need to refine the five parameters for every particle image during the projection matching process. In this paper, we focus on the refinement of the three Euler angles, the proposed algorithm can be easily extended to refine all the five parameters.

The projection matching refinement methods, first proposed in [23], minimize the difference between projections and particle images. The most common algorithm of the projection matching refinement methods starts from a coarse initial angular increment (the second Euler angle $\phi$ ), obtaining quasi-even distributions of projection directions on the unit sphere $\mathbb{S}^{2}$. Then it calculates 
the projection images using these directions. After that, it computes the similarity between every projection and particle image using Euclidean distance or cross-correlation in order to find the best matched projection for every particle image. The five parameters of the particle image then can be refined according to the best matched projection. After that, a new map is reconstructed using the particle images with refined parameters. The new map is utilized to perform new projection matching with a finer angular increment. The algorithm has been realized in some cryo-EM softwares, including EMAN [21] and Xmipp [25]. Another two refinement algorithms, one developed in the software package Frealign [15], using the Powell optimization algorithm, the other published in [18], utilizing the Nelder-Mead simplex minimization algorithm [22], both maximize the correlation coefficients in the Fourier space.

One of the main obstacles in the cryo-EM SPM image processing is the extremely low SNR of the particles, which results in the errors in the estimation of orientations. Hence, the main task of the orientation refinement is to overcome the affect of the noise as much as possible so as to reduce the errors of the orientations. Recently, the development of the wavelet based methods that find sparse solutions by minimizing $\ell_{1}$-norm of wavelet orthonormal bases or wavelet tight frame coefficients has obtained successful applications in the image restorations $[2,3,4,9,10,11]$ and cryo-EM SPM 3D reconstruction [19]. That motivates us to involve this idea in the refinement of cryo-EM SPM map. In this paper, we explore the wavelet orthonormal bases for its convenience in the theoretical analysis.

\section{Orthonormal Wavelet Based Alternative Iteration Method}

The proposed algorithm utilizes the sparse approximation of the 3D density map under the wavelet orthonormal bases and performs the orientation refinement and 3D reconstruction alternatively. In order to do 3D reconstruction, we need to compute the discretized X-ray transform and back-projection.

Let $f \in \mathbb{R}^{n^{3}}$ denote the vector of the 3D density map with dimension $n \times n \times n, g_{i} \in \mathbb{R}^{n^{2}}(i=1, \ldots, m)$ be the measured 2D particle images. Matrix $P_{i} \in \mathbb{R}^{n^{2} \times n^{3}}$ is the discretized form of the group of 3D X-ray transforms $\mathcal{P}$ for the orientation $\left(\theta_{i}, \phi_{i}, \psi_{i}\right)$ and correspondingly $\left[P_{1}^{\top}, \ldots, P_{m}^{\top}\right]$ is the discretized form of the back-projection operator $\mathcal{P}^{*}$. Let

$$
A=\left[P_{1}^{\top}, \ldots, P_{m}^{\top}\right]^{\top}, \quad g=\left[g_{1}^{\top}, \ldots, g_{m}^{\top}\right]^{\top},
$$

we obtain the linear system:

$$
A f=g-\epsilon,
$$

where $\epsilon$ is the unknown noise. Since the size of matrix $A$ and $A^{\top}$ are huge for the cryo-EM SPM problem, it is not efficient to save the matrix in the computer's memory. In practice, we only compute and save $A f$ and $A^{\top} g$. Figure 1 shows a $2 \mathrm{D}$ example for computing $A f$ and $A^{\top} g$. Suppose that $l$ is a line with a given projection direction coming across the map $f$ and intersecting it with some pixels in $f$. We simply sum these pixel values to obtain the discrete 
approximation of X-ray transform, as Figure 1 (a) shows. We loop all the directions and lines to obtain $A f$. In order to compute $u=A^{\top} g$, for each pixel $u_{i}$ of $u$, we sum these pixel values of $g$ whose back-projection directions come across $u_{i}$, as shown in Figure 1 (b). We loop all the pixels in $u_{i}$ to obtain $u$.

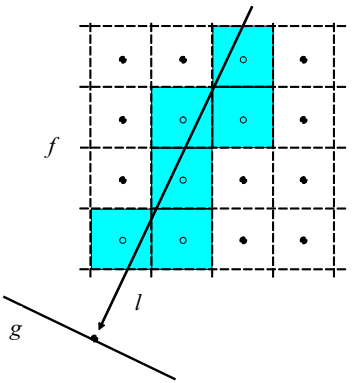

(a)

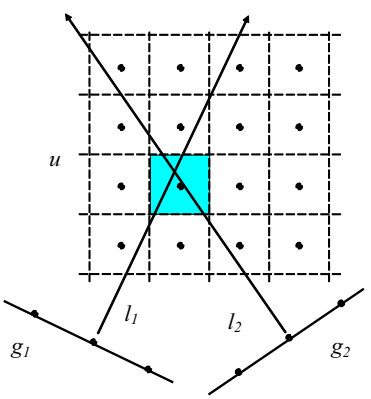

(b)

Figure 1. (a) Discretized 2D X-ray tranform; (b) Discretized 2D back projection.

In order to reconstruct a 3D density map from its 2D cryo-EM SPM images and refine the Euler angles, we propose the following minimization problem:

$$
\min _{\Lambda, f} E(f, \Lambda)=\frac{\kappa}{2} \sum_{i=1}^{m}\left\|P_{i}\left(\alpha_{i}\right) f-g_{i}\right\|_{2}^{2}+\|\operatorname{diag}(\lambda) W f\|_{1},
$$

s.t. $\alpha_{i} \in \Omega_{i}, i=1, \ldots, m$,

where $\kappa>0, \lambda \in \mathbb{R}_{+}^{n^{3}}, \Lambda=\left(\alpha_{1}, \ldots, \alpha_{m}\right)^{\top}, \alpha_{i}=\left(\theta_{i}, \phi_{i}, \psi_{i}\right)^{\top}$, and $\Omega_{i} \subset \mathbb{R}^{3}$, $i=1, \ldots, m$ are bounded closed convex subsets. $W$ is the orthonormal wavelet decomposition matrix satisfying $W^{\top} W=I, W W^{\top}=I$. Suppose that we have obtained the initial estimations of $\Lambda$ and $f$, denoted by $\Lambda^{0}$ and $f^{0}$. Our goal is to refine these parameters $\Lambda$ and $f$ so as to improve the resolution of the $3 \mathrm{D}$ map. Hence, we propose to solve the following alterative minimization problems:

$$
\begin{aligned}
\alpha_{i}^{k+1} & =\arg \min _{\alpha_{i} \in \Omega_{i}} \frac{\kappa}{2}\left\|P_{i}\left(\alpha_{i}\right) f^{k}-g_{i}\right\|_{2}^{2}, \quad i=1, \ldots, m ; \\
f^{k+1} & =\arg \min _{f} \frac{\kappa}{2} \sum_{i=1}^{m}\left\|P_{i}\left(\alpha_{i}^{k+1}\right) f-g_{i}\right\|_{2}^{2}+\|\operatorname{diag}(\lambda) W f\|_{1} .
\end{aligned}
$$

The subproblem (2.3) is a nonlinear least square problem and can be solved using the Levenberg-Marquardt algorithm. Let $F_{i}^{k}\left(\alpha_{i}\right):=P_{i}\left(\alpha_{i}\right) f^{k}-g_{i} \in \mathbb{R}^{n^{2}}$, matrix $J_{i}^{k}\left(\alpha_{i}\right) \in \mathbb{R}^{n^{2} \times 3}$ is the Jacobian matrix of $F_{i}^{k}\left(\alpha_{i}\right)$ defined by

$$
J_{i}^{k}\left(\alpha_{i}\right)=\left(\begin{array}{ccc}
\frac{\partial F_{i, 1}^{k}}{\partial \theta_{i}} & \frac{\partial F_{i, 1}^{k}}{\partial \phi_{i}} & \frac{\partial F_{i, 1}^{k}}{\partial \psi_{i}} \\
\vdots & \ddots & \vdots \\
\frac{\partial F_{i, n^{2}}^{k}}{\partial \theta_{i}} & \frac{\partial F_{i, n^{2}}^{k}}{\partial \phi_{i}} & \frac{\partial F_{i, n^{2}}^{k}}{\partial \psi_{i}}
\end{array}\right) .
$$


We consider the linearized problem of (2.3) at the point $\alpha_{i}^{k}$

$$
s_{i}^{k}=\arg \min _{s_{i}} \frac{1}{2}\left\|F_{i}^{k}\left(\alpha_{i}^{k}\right)+J_{i}^{k}\left(\alpha_{i}^{k}\right) s_{i}\right\|_{2}^{2} .
$$

The Levenberg-Marquardt algorithm gives

$$
s_{i}^{k}=-\left[J_{i}^{k}\left(\alpha_{i}^{k}\right)^{\top} J_{i}^{k}\left(\alpha_{i}^{k}\right)+\mu_{i}^{k} I\right]^{-1} J_{i}^{k}\left(\alpha_{i}^{k}\right)^{\top} F_{i}^{k}\left(\alpha_{i}^{k}\right),
$$

and the updating scheme of $\alpha_{i}$ is given by

$$
\alpha_{i}^{k+1}=\alpha_{i}^{k}-\sigma_{i}^{k}\left[J_{i}^{k}\left(\alpha_{i}^{k}\right)^{\top} J_{i}^{k}\left(\alpha_{i}^{k}\right)+\mu_{i}^{k} I\right]^{-1} J_{i}^{k}\left(\alpha_{i}^{k}\right)^{\top} F_{i}^{k}\left(\alpha_{i}^{k}\right) .
$$

The subproblem (2.4) can be solved by the iterative soft-thresholding algorithm:

$$
f^{k+1}=W^{\top} T_{\lambda / \kappa} W\left(f^{k}-\kappa \sigma_{f}^{k} \sum_{i=1}^{m} P_{i}\left(\alpha_{i}^{k+1}\right)^{\top} F_{i}^{k}\left(\alpha_{i}^{k+1}\right)\right),
$$

where $T_{\lambda / \kappa}$ is defined by

$$
T_{\lambda / \kappa}\left(\left[u_{1}, u_{2}, \ldots, u_{n^{3}}\right]^{\top}\right)=\left[t_{\lambda_{1} / \kappa}\left(u_{1}\right), t_{\lambda_{2} / \kappa}\left(u_{2}\right), \ldots, t_{\lambda_{n^{3}} / \kappa}\left(u_{n^{3}}\right)\right]^{\top}
$$

with the soft-thresholding operator $t_{\lambda_{i} / \kappa}\left(u_{i}\right)=\operatorname{sgn}\left(u_{i}\right) \max \left(\left|u_{i}\right|-\lambda_{i} / \kappa, 0\right)$. Using (2.1) the iteration scheme (2.5) can be reformulated as

$$
f^{k+1}=W^{\top} T_{\lambda / \kappa} W\left(f^{k}-\kappa \sigma_{f}^{k}\left(A^{k+1}\right)^{\top}\left(A^{k+1} f^{k}-g\right)\right) .
$$

The stepsizes $\sigma_{i}^{k}$ and $\sigma_{f}^{k}$ are choosen using the following Armijo rule.

Definition 1. Armijo rule [1]. Given the descent direction $d^{k}$ of function $E(x), s, \beta$ and $\rho$ with $s>0,0<\beta<1$, and $0<\rho<1$, choosing $m_{k}$ as the first nonnegative integer $m$ satisfying

$$
E\left(x^{k}\right)-E\left(x^{k}+\beta^{m} s d^{k}\right) \geq-\rho \beta^{m} s \nabla E\left(x^{k}\right)^{\top} d^{k},
$$

then set the stepsize $\sigma_{k}=\beta^{m_{k}} s$.

Then the algorithm solving the orientation refinement problem (2.2) is proposed as follows.

Algorithm 1 Given $\kappa>0,0<\rho<1, s>0,0<\beta<1, \alpha_{i}^{0} \in \Omega_{i}, \mu_{i}^{0} \geq 0$, $i=1, \ldots, m$ and $f^{0} \in \mathbb{R}^{n^{3}}$. For $k=0,1,2, \ldots$,

1. for $i=1, \ldots, m$

(1) $s_{i}^{k}=-\left[J_{i}^{k}\left(\alpha_{i}^{k}\right)^{\top} J_{i}^{k}\left(\alpha_{i}^{k}\right)+\mu_{i}^{k} I\right]^{-1} J_{i}^{k}\left(\alpha_{i}^{k}\right)^{\top} F_{i}^{k}\left(\alpha_{i}^{k}\right)$.

(2) Choose a stepsize $\sigma_{i}^{k}=\beta^{m_{k}} s$ satisfying the Armijo rule

$$
\left\|F_{i}^{k}\left(\alpha_{i}^{k}+\sigma_{i}^{k} s_{i}^{k}\right)\right\|^{2} \leq\left\|F_{i}^{k}\left(\alpha_{i}^{k}\right)\right\|^{2}+\rho \sigma_{i}^{k}\left[J_{i}^{k}\left(\alpha_{i}^{k}\right)^{\top} F_{i}^{k}\left(\alpha_{i}^{k}\right)\right]^{\top} s_{i}^{k} .
$$


(3) If $\alpha_{i}^{k}+\sigma_{i}^{k} s_{i}^{k} / \beta \notin \Omega_{i}$, then let $\mu_{i}^{k+1}=4 \mu_{i}^{k}, \alpha_{i}^{k+1}=\alpha_{i}^{k}, i=i+1$, go to (1).

(4) $\alpha_{i}^{k+1}=\alpha_{i}^{k}+\sigma_{i}^{k} s_{i}^{k}, \mu_{i}^{k+1}=0.5 \mu_{i}^{k}, i=i+1$.

2. $d_{f}^{k}=-\kappa\left(A^{k+1}\right)^{\top}\left(A^{k+1} f^{k}-g\right)$

(1) Choose a stepsize $\sigma_{f}^{k}>0$ satisfying the Armijo rule

$$
E\left(W^{\top} T_{\lambda / \kappa} W\left(f^{k}+\sigma_{f}^{k} d_{f}^{k}\right), \Lambda^{k+1}\right) \leq E\left(f^{k}, \Lambda^{k+1}\right)-\rho \sigma_{f}^{k} d_{f}^{k^{\top}} d_{f}^{k} .
$$

3. $f^{k+1}=W^{\top} T_{\lambda / \kappa} W\left(f^{k}+\sigma_{f}^{k} d_{f}^{k}\right)$.

In the implement of the Algorithm 1, the jacobian matrix $J_{i}^{k}\left(\alpha_{i}\right)$ is approximately calculated by employing the finite difference scheme. For example, in order to compute $\frac{\partial F_{i, 1}}{\partial \theta_{i}}$, we have

$$
\frac{\partial F_{i, 1}}{\partial \theta_{i}} \approx \frac{F_{i, 1}\left(\theta_{i}^{k}+h^{k}, \phi_{i}^{k}, \psi_{i}^{k}\right)-F_{i, 1}\left(\theta_{i}^{k}, \phi_{i}^{k}, \psi_{i}^{k}\right)}{h^{k}} .
$$

\section{Convergence Analysis}

In this section, we analyze the convergence of the proposed algorithm. The result is given by the following theorem.

Theorem 1. Let $\Omega_{i} \subset \mathbb{R}^{3}, i=1, \ldots, m$ be bounded closed convex subsets, and $\left\{A^{k^{\top}} A^{k}\right\}$ be a nonsingular matrix sequence for $\alpha_{i} \in \Omega_{i}, i=1, \ldots, m$ and uniformly bounded. Then we have

(1) the sequences $\left\{\alpha_{i}^{k}\right\}, i=1, \ldots, m$ and $\left\{f^{k}\right\}$ generated by Algorithm 1 have convergent subsequences, denoted by $\left\{\alpha_{i}^{k}\right\}_{\mathcal{K}}, i=1, \ldots, m$, and $\left\{f^{k}\right\}_{\mathcal{K}}$, where $\mathcal{K} \subseteq\{0,1, \ldots$,$\} .$

(2) Let $\lim _{\substack{k \rightarrow \infty \\ k \in \mathcal{K}}} \alpha_{i}^{k}=a_{i}^{*}, i=1, \ldots, m ; \lim _{\substack{k \rightarrow \infty \\ k \in \mathcal{K}}} f^{k}=f^{*}$. If there exists two positive scalars $c_{1}, c_{2}$ and $\mu_{i}^{k}>0$, such that

$c_{1}\|x\|^{2} \leq x^{\top}\left[J_{i}^{k}\left(\alpha_{i}^{k}\right)^{\top} J_{i}^{k}\left(\alpha_{i}^{k}\right)+\mu_{i}^{k} I\right]^{-1} x \leq c_{2}\|x\|^{2}, \quad \forall x \in \mathbb{R}^{3}, i=1, \ldots, m$

for all $k$, then $\left(f^{*}, \alpha_{1}^{*}, \ldots, \alpha_{m}^{*}\right)$ is a stationary point.

Proof. (1). For $i=1,2, \ldots, m$, since $\left\{\alpha_{i}^{k}\right\} \subset \Omega_{i}$, then there exists a convergent subsequence $\left\{\alpha_{i}^{k}\right\}_{\mathcal{K}_{1}}\left(\mathcal{K}_{1} \subseteq\{0,1, \ldots\},\right)$.

Taking the spectral norm of the matrix, we have

$$
\|I\|=1, \quad\|W\|^{2}=\left\|W^{\mathrm{T}} W\right\|=1,\|W\|=\left\|W^{\mathrm{T}}\right\|=1,
$$

and according to the result in [7], $T_{\lambda}$ is nonexpansive, then $\forall u, v \in \mathbb{R}^{n^{3}}$,

$$
\begin{aligned}
\left\|W^{\top} T_{\lambda} W u-W^{\top} T_{\lambda} W v\right\| & =\left\|W^{\top}\left(T_{\lambda} W u-T_{\lambda} W v\right)\right\| \\
& \leq\left\|W^{\top}\right\|\left\|T_{\lambda} W u-T_{\lambda} W v\right\| \\
& \leq\|W u-W v\|=\|u-v\| .
\end{aligned}
$$

By taking $v=0$, we have $\left\|W^{\top} T_{\lambda} W u\right\| \leq\|u\|$. 
Since the matrix $A^{k^{\top}} A^{k}$ is nonsingular and uniformly bounded, there exist $\sigma_{M}, \sigma_{m}>0$ such that $0<\left\|A^{k}\right\|<\sigma_{M}$, and for all $k=1, \ldots$, the minimal singular value $\sigma_{\min }\left(A^{k}\right)>\sigma_{m}$. Take $\frac{1}{2 \sigma_{M}^{2}}<\kappa<\frac{1}{\sigma_{M}^{2}}$, then

$$
0<\left\|I-\kappa A^{k^{\top}} A^{k}\right\|<1-0.5 \sigma_{m}^{2} / \sigma_{M}^{2} .
$$

Denote $\omega=1-0.5 \sigma_{m}^{2} / \sigma_{M}^{2}$, then

$$
\begin{aligned}
\left\|f^{k+1}\right\| & =\left\|W^{\top} T_{\lambda / \kappa} W\left[\left(I-\kappa A^{k^{\top}} A^{k}\right) f^{k}+\kappa A^{k^{\top}} g\right]\right\| \\
& \leq\left\|\left(I-\kappa A^{k^{\top}} A^{k}\right) f^{k}+\kappa A^{k^{\top}} g\right\| \leq \omega\left\|f^{k}\right\|+\kappa \sigma_{M}\|g\| \\
& \leq \omega^{k+1}\left\|f^{0}\right\|+\kappa \sigma_{M}\|g\| \frac{1-\omega^{k+1}}{1-\omega} \leq\left\|f^{0}\right\|+\frac{\|g\|}{\sigma_{M}(1-\omega)} .
\end{aligned}
$$

Therefore, there exists a convergent subsequence $\left\{f^{k}\right\}_{\mathcal{K} \subseteq \mathcal{K}_{1}}$.

(2). From the left inequality of (3.1) we can deduce that $\left[J_{i}^{k}\left(\alpha_{i}^{k}\right)^{\top} J_{i}^{k}\left(\alpha_{i}^{k}\right)+\right.$ $\left.\mu_{i}^{k} I\right]^{-1}$ is positive definite, and $\left[J_{i}^{k}\left(\alpha_{i}^{k}\right)^{\top} F_{i}^{k}\left(\alpha_{i}^{k}\right)\right]^{\top} s_{i}^{k}<0$, the Armijo rule (2.6) implies that

$$
\left\|F_{i}^{k}\left(\alpha_{i}^{k}+\sigma_{i}^{k} s_{i}^{k}\right)\right\| \leq\left\|F_{i}^{k}\left(\alpha_{i}^{k}\right)\right\|
$$

then for $i=1, \ldots, m$ we have

$$
E\left(f^{k}, \alpha_{1}^{k+1}, \ldots, \alpha_{i}^{k+1}, \alpha_{i+1}^{k}, \ldots, \alpha_{m}^{k}\right) \leq E\left(f^{k}, \alpha_{1}^{k+1}, \ldots, \alpha_{i}^{k}, \alpha_{i+1}^{k}, \ldots, \alpha_{m}^{k}\right) .
$$

The Armijo rule (2.7) implies that

$$
E\left(f^{k+1}, \alpha_{1}^{k+1}, \ldots, \alpha_{m}^{k+1}\right) \leq E\left(f^{k}, \alpha_{1}^{k+1}, \ldots, \alpha_{m}^{k+1}\right) .
$$

Using (3.2) and (3.3), we obtain

$$
E\left(f^{k+1}, \alpha_{1}^{k+1}, \ldots, \alpha_{m}^{k+1}\right) \leq E\left(f^{k}, \alpha_{1}^{k}, \ldots, \alpha_{m}^{k}\right) .
$$

Hence $\left\{E\left(f^{k}, \alpha_{1}^{k}, \ldots, \alpha_{m}^{k}\right)\right\}$ is nonincreasing. Since $\left\{\left(f^{k}, \alpha_{1}^{k}, \ldots, \alpha_{m}^{k}\right)\right\}_{k \in \mathcal{K}}$ converges to $\left(f^{*}, \alpha_{1}^{*}, \ldots, \alpha_{m}^{*}\right)$ and $E$ is continuous, we have

$$
\lim _{\substack{k \rightarrow \infty \\ k \in \mathcal{K}}} E\left(f^{k}, \alpha_{1}^{k}, \ldots, \alpha_{m}^{k}\right)=E\left(f^{*}, \alpha_{1}^{*}, \ldots, \alpha_{m}^{*}\right) .
$$

Then from (2.6) and (2.7) we have

$$
\begin{aligned}
& \left\{\sigma_{i}^{k}\left[J_{i}^{k}\left(\alpha_{i}^{k}\right)^{\top} F_{i}^{k}\left(\alpha_{i}^{k}\right)\right]^{\top} s_{i}^{k}\right\} \rightarrow 0, \quad i=1, \ldots, m, \\
& \left\{\sigma_{f}^{k} d_{f}^{k^{\top}} d_{f}^{k}\right\} \rightarrow 0 .
\end{aligned}
$$

Suppose that $\left\{s_{i}^{k}\right\}_{\mathcal{K}} \nrightarrow 0$ and $\left\{d_{f}^{k}\right\}_{\mathcal{K}} \nrightarrow 0$. By possibly restricting to subsequences of $\left\{s_{i}^{k}\right\}_{\mathcal{K}}$ and $\left\{d_{f}^{k}\right\}_{\mathcal{K}}$, we assume that for some $\delta>0,\left\|s_{i}^{k}\right\| \geq \delta$, $\left\|d_{f}^{k}\right\| \geq \delta$, for all $k \in \mathcal{K}$. Then, from (3.4) and (3.5), we have $\left\{\sigma_{i}^{k}\right\}_{\mathcal{K}} \rightarrow 0$, $i=1, \ldots, m$ and $\left\{\sigma_{f}^{k}\right\}_{\mathcal{K}} \rightarrow 0$. By the Definition 1 of the Armijo rule, and the Algorithm 1, we have for $i=1, \ldots, m$

$$
\begin{aligned}
& \left\|F_{i}^{k}\left(\alpha_{i}^{k}\right)\right\|^{2}-\left\|F_{i}^{k}\left(\alpha_{i}^{k}+\left(\sigma_{i}^{k} / \beta\right) s_{i}^{k}\right)\right\|^{2} \\
& \quad<-\rho\left(\sigma_{i}^{k} / \beta\right)\left[J_{i}^{k}\left(\alpha_{i}^{k}\right)^{\top} F_{i}^{k}\left(\alpha_{i}^{k}\right)\right]^{\top} s_{i}^{k}, \quad \forall k \in \mathcal{K},
\end{aligned}
$$


where $\alpha_{i}^{k}+\left(\sigma_{i}^{k} / \beta\right) s_{i}^{k} \in \Omega_{i}$. Denote $p_{i}^{k}=s_{i}^{k} /\left\|s_{i}^{k}\right\|, \bar{\sigma}_{i}{ }^{k}=\sigma^{k}\left\|s_{i}^{k}\right\| / \beta$, then from (3.6) we have

$$
\frac{\left\|F_{i}^{k}\left(\alpha_{i}^{k}\right)\right\|^{2}-\left\|F_{i}^{k}\left(\alpha_{i}^{k}+{\overline{\sigma_{i}}}^{k} p_{i}^{k}\right)\right\|^{2}}{{\overline{\sigma_{i}}}^{k}}<-\rho\left[J_{i}^{k}\left(\alpha_{i}^{k}\right)^{\top} F_{i}^{k}\left(\alpha_{i}^{k}\right)\right]^{\top} p_{i}^{k}, \quad \forall k \in \mathcal{K} .
$$

By using the mean value theorem, this relation becomes

$-\left[J_{i}^{k}\left(\alpha_{i}^{k}+{\tilde{\sigma_{i}}}^{k} p_{i}^{k}\right)^{\top} F_{i}^{k}\left(\alpha_{i}^{k}+{\tilde{\sigma_{i}}}^{k} p_{i}^{k}\right)\right]^{\top} p_{i}^{k}<-\rho\left[J_{i}^{k}\left(\alpha_{i}^{k}\right)^{\top} F_{i}^{k}\left(\alpha_{i}^{k}\right)\right]^{\top} p_{i}^{k}, \quad \forall k \in \mathcal{K}$,

where $\tilde{\sigma}_{i}{ }^{k} \in\left[0, \bar{\sigma}_{i}{ }^{k}\right]$. From the right inequality of (3.1) we can deduce that $\left\|\left[J_{i}^{k}\left(\alpha_{i}^{k}\right)^{\top} J_{i}^{k}\left(\alpha_{i}^{k}\right)+\mu_{i}^{k} I\right]^{-1}\right\| \leq c_{2}$, then

$$
\begin{aligned}
\sigma_{k}\left\|s_{i}^{k}\right\| & \leq \sigma_{k}\left\|\left[J_{i}^{k}\left(\alpha_{i}^{k}\right)^{\top} J_{i}^{k}\left(\alpha_{i}^{k}\right)+\mu_{i}^{k} I\right]^{-1}\right\|\left\|J_{i}^{k}\left(\alpha_{i}^{k}\right)^{\top} F_{i}^{k}\left(\alpha_{i}^{k}\right)\right\| \\
& \leq \sqrt{\sigma_{k}}\left\|\left[J_{i}^{k}\left(\alpha_{i}^{k}\right)^{\top} J_{i}^{k}\left(\alpha_{i}^{k}\right)+\mu_{i}^{k} I\right]^{-1}\right\|\left(\frac{\left\|\sigma_{k}\left[J_{i}^{k}\left(\alpha_{i}^{k}\right)^{\top} F_{i}^{k}\left(\alpha_{i}^{k}\right)\right]^{\top} s_{i}^{k}\right\|}{c_{1}}\right)^{1 / 2} .
\end{aligned}
$$

Using (3.4) we have $\left\{\bar{\sigma}_{i}{ }^{k}\right\}_{\mathcal{K}} \rightarrow 0$, and since $\left\|p_{i}^{k}\right\|=1, \forall k \in \mathcal{K}$, there exists a subsequence $\left\{p_{i}^{k}\right\}_{\overline{\mathcal{K}} \subseteq \mathcal{K}}$ such that

$$
\left\{p_{i}^{k}\right\}_{\overline{\mathcal{K}}} \rightarrow p_{i}^{*}, \quad\left\|p_{i}^{*}\right\|=1
$$

Taking limits in the equation (3.7) we obtain

$$
-\left[J_{i}\left(\alpha_{i}^{*}\right)^{\top} F_{i}\left(\alpha_{i}^{*}\right)\right]^{\top} p_{i}^{*} \leq-\rho\left[J_{i}\left(\alpha_{i}^{*}\right)^{\top} F_{i}\left(\alpha_{i}^{*}\right)\right]^{\top} p_{i}^{*},
$$

or

$$
0 \leq(1-\rho)\left[J_{i}\left(\alpha_{i}^{*}\right)^{\top} F_{i}\left(\alpha_{i}^{*}\right)\right]^{\top} p_{i}^{*} .
$$

Since $0<\rho<1$, it follows that

$$
\left[J_{i}\left(\alpha_{i}^{*}\right)^{\top} F_{i}\left(\alpha_{i}^{*}\right)\right]^{\top} p_{i}^{*} \geq 0 .
$$

On the other hand,

$$
\left[J_{i}^{k}\left(\alpha_{i}^{k}\right)^{\top} F_{i}^{k}\left(\alpha_{i}^{k}\right)\right]^{\top} p_{i}^{k}=\frac{\left[J_{i}^{k}\left(\alpha_{i}^{k}\right)^{\top} F_{i}^{k}\left(\alpha_{i}^{k}\right)\right]^{\top} s_{i}^{k}}{\left\|s_{i}^{k}\right\|}<0 .
$$

By taking the limits as $k \in \overline{\mathcal{K}}, k \rightarrow \infty$,

$$
\left[J_{i}\left(\alpha_{i}^{*}\right)^{\top} F_{i}\left(\alpha_{i}^{*}\right)\right]^{\top} p_{i}^{*}<0,
$$

which arrives at a contradiction. Hence, we have $\left\{s_{i}^{k}\right\}_{\mathcal{K}} \rightarrow 0$, and it follows that $J_{i}\left(\alpha_{i}^{*}\right)^{\top} F_{i}\left(\alpha_{i}^{*}\right)=0$ for $i=1, \ldots, m$.

From the boundedness of $\left\|A^{k+1}\right\|$ and $\left\|A^{k+1} f^{k}-g\right\|$ we can deduce that $d_{f}^{k}$ is bounded. And then using the Armijo rule (2.7) we can prove that $d_{f}^{*}=0$ similarly. Hence, $\left(f^{*}, \alpha_{1}^{*}, \ldots, \alpha_{m}^{*}\right)$ is a stationary point of $E(f, \Lambda)$. Part $(2)$ of the theorem is proved. 
Based on the discretization method of the X-ray transform we used, the matrix $P_{i}$ is full row rank since no two lines can go through the same set of voxels. For the cryo-EM SPM problem, the number of orientations is normally far more than the number of images, that is $m \gg n$. When the orientations of the images are distributed even uniformly on the unit sphere, $A^{k^{\top}} A^{k}$ is nonsingular. The uniformly boundedness of $A^{k^{\top}} A^{k}$ can be satisfied if $A^{k}$ is uniformly bounded. In fact, from the discretization method of the X-ray transform we proposed, the elements of $A^{k}$ are 1 or 0 . Therefore, for $m$ orientations and $n$ images, the Frobenius norm of $\left\|A^{k}\right\|_{F}$ satisfies $\left\|A^{k}\right\|_{F} \leq \sqrt{m n^{3}}$, which means $A^{k}$ is uniformly bounded. Finally, the condition (3.1) can be satisfied by Algorithm 1 by adjusting the parameter $\mu_{i}^{k}$ at each iteration.

\section{Numerical Experiments}

In this experiment, we simulate the cryo-EM SPM particle images using the E. coli ribosome crystal structure 3I1M.pdb ${ }^{1}$ and $3 \mathrm{I} 1 \mathrm{~N} . \mathrm{pdb}^{2}$. A 3D density map of E. coli ribosome with pixel size $2.82 \AA$ and volume size $131 \times 131 \times 131$ is generated by first combining the two crystal structure into one and then filtering it using Gaussian convolution. After that, 5000 simulated particle images with size $131 \times 131$ pixels are generated from this $3 \mathrm{D}$ density map using randomly orientations, part of which are shown in Figure 2.

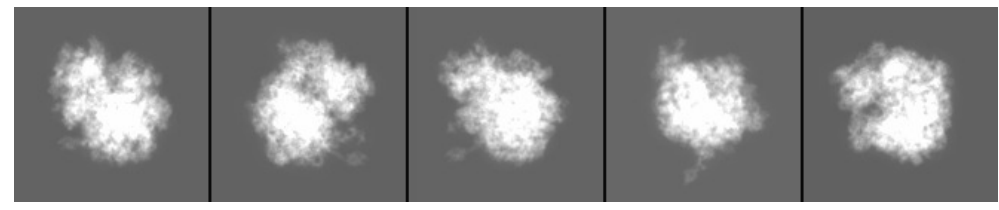

Figure 2. Simulated E. coli ribosome cryo-EM SPM particle images.

Each orientation for one image is saved as three Euler angles $(\theta, \phi, \psi)$. In order to construct the dataset for the proposed algorithm, we disturb the set of Euler angles to produce inaccurate Euler angles $(\tilde{\theta}, \tilde{\phi}, \tilde{\psi})$, that is $\left|\tilde{\theta}_{i}-\theta_{i}\right|<$ $\delta,\left|\tilde{\phi}_{i}-\phi_{i}\right|<\delta$ and $\left|\tilde{\psi}_{i}-\psi_{i}\right|<\delta$ for $i=1, \ldots, 5000$. We takes $\delta=1^{\circ}, 3^{\circ}, 9^{\circ}, 21^{\circ}$ and $39^{\circ}$ respectively to obtain five sets of inaccurate Euler angles $\Lambda_{i}^{0}, i=$ $1, \ldots, 5$ and these five sets of Euler angles are used to reconstruct five errored 3D maps $f_{i}^{0}, i=1, \ldots, 5$. Each pair of $\left(\Lambda_{i}^{0}, f_{i}^{0}\right)$ are used to test the proposed algorithm. The proposed algorithm is implemented using Haar wavelet orthonormal base, parallelized using MPI (message passing interface), and run on the computer cluster ${ }^{3}$ with totally 100 processes. These test results are shown in Table 1. The mean square error (MSE) is used to evaluate the algorithm which defines as follows

$$
\operatorname{MSE}\left(\Lambda, \Lambda^{k}\right)=\frac{1}{m}\left\|\Lambda-\Lambda^{k}\right\|_{F},
$$

\footnotetext{
1 http://www.rcsb.org/pdb/explore.do?structureId=3i1m

2 http://www.rcsb.org/pdb/explore.do?structureId=3I1N

3 Computer cluster "LSSC3" in ICMSEC at CAS with totally 282 nodes annd 24 GB memory each
} 
where $\|\cdot\|_{F}$ is the Frobenius norm, $\Lambda$ and $\Lambda^{k}$ are the correct Euler angles and the refinement results at the iteration $k$, respectively. Define InitMSE $=$ $\operatorname{MSE}\left(\Lambda, \Lambda^{0}\right)$ as the initial error.

Table 1. Orientation refinement results for the simulated cryo-EM SPM particle images.

\begin{tabular}{llllll}
\hline clean image & $1^{\circ}$ & $3^{\circ}$ & $9^{\circ}$ & $21^{\circ}$ & $39^{\circ}$ \\
\hline InitMSE & 0.02451 & 0.0733 & 0.2213 & 0.5137 & 0.9559 \\
MSE & 0.01096 & 0.0169 & 0.0250 & 0.0554 & 0.2408 \\
iterations & 30 & 38 & 48 & 48 & 57 \\
\hline
\end{tabular}

From Table 1 we can conclude that the algorithm improve the errors of the Euler angles, even for the big angles errors $39^{\circ}$. The output reconstructions are shown in Figure 3, from which we can see that the resolutions of the 3D maps are improved apparently by the proposed algorithm.

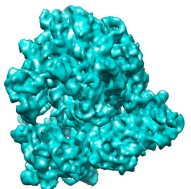

$\left(a_{1}\right)$

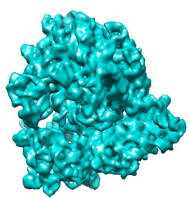

$\left(a_{2}\right)$

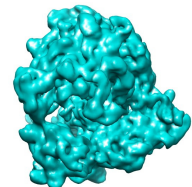

$\left(b_{1}\right)$

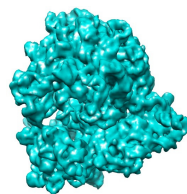

$\left(b_{2}\right)$

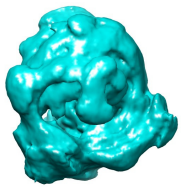

$\left(c_{1}\right)$

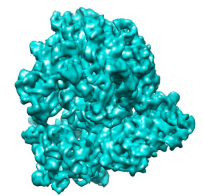

$\left(c_{2}\right)$

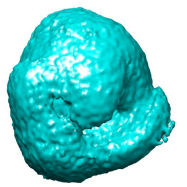

$\left(d_{1}\right)$

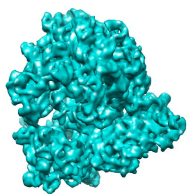

$\left(d_{2}\right)$

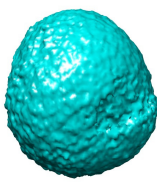

$\left(e_{1}\right)$

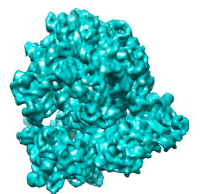

$\left(e_{2}\right)$

Figure 3. The comparisons of the initial maps (first row) and the refinement maps (second row) using the proposed algorithm. The maps from column (a) to column (e) show the cases for $1^{\circ}, 3^{\circ}, 9^{\circ}, 21^{\circ}$ and $39^{\circ}$ respectively.

Since cryo-EM SPM particle images have extremely low SNR, in our second experiment, Gaussian white noise is added on each image to obtain noisy image with $\mathrm{SNR}=0.1$. Part of these simulated noisy particle images are shown in Figure 4 .

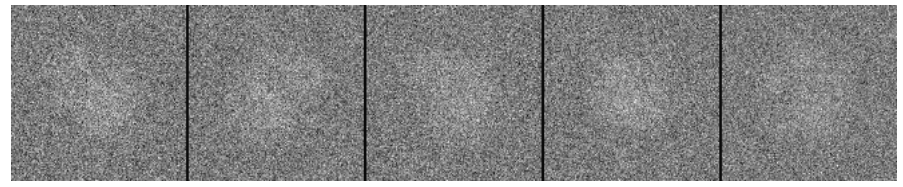

Figure 4. Simulated E. coli ribosome cryo-EM SPM particle images with SNR $=0.1$.

We run the proposed algorithm for the five pairs of initial values $\left(\Lambda_{i}^{0}, f_{i}^{0}\right), i=$ $1, \ldots, 5$ again for the noisy images. Table 2 gives the results. We can see that 
Table 2. Orientation refinement results for the simulated noisy particle images.

\begin{tabular}{llllll}
\hline $\mathrm{SNR}=0.1$ & $1^{\circ}$ & $3^{\circ}$ & $9^{\circ}$ & $21^{\circ}$ & $39^{\circ}$ \\
\hline InitMSE & 0.02451 & 0.0733 & 0.2213 & 0.5137 & 0.9559 \\
MSE & 0.02384 & 0.0550 & 0.1007 & 0.1911 & 0.5258 \\
iterations & 12 & 15 & 33 & 42 & 49 \\
\hline
\end{tabular}

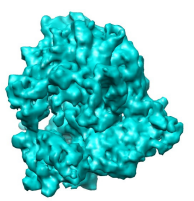

$\left(a_{1}\right)$

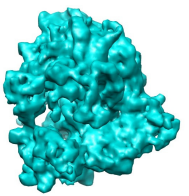

$\left(a_{2}\right)$

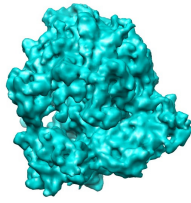

$\left(b_{1}\right)$

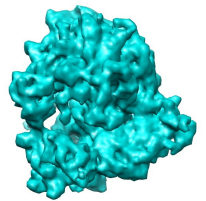

$\left(b_{2}\right)$

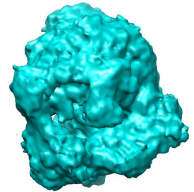

$\left(c_{1}\right)$

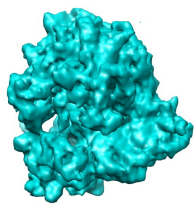

$\left(c_{2}\right)$

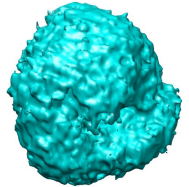

$\left(d_{1}\right)$

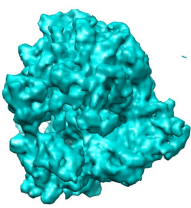

$\left(d_{2}\right)$

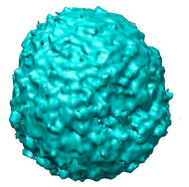

$\left(e_{1}\right)$

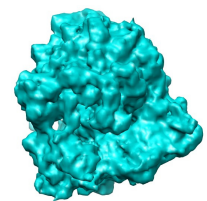

$\left(e_{2}\right)$

Figure 5. The comparisons of the initial maps (first row) and the refinement maps (second row) using the proposed algorithm for the noisy images. The maps from column (a) to column (e) show the cases for $1^{\circ}, 3^{\circ}, 9^{\circ}, 21^{\circ}$ and $39^{\circ}$ respectively.

the errors can still be improved even for the worst case $39^{\circ}$. The reconstructed maps for the refinement Euler angles are given in Figure 5. We can conclude that for the noisy images, the reconstructed maps using the proposed algorithm have obtained improved resolutions compared with the initial maps.

\section{Conclusions}

In this paper we have proposed the alternative iteration orientation refinement method combining the Levenberg-Marquardt algorithm and wavelet orthonormal bases based iterative soft-thresholding. The convergence analysis and numerical experiments show that the proposed algorithm is effective and efficient for the simulated cryo-EM SPM particle images. For the true cryo-EM SPM experimental particle images, since the noise in the images is a combination of several different noise sources coming from the imaging process, the algorithm will be improved in the future to deal with the non-Gaussian noise images.

\section{Acknowledgments}

The authors would like to thank the reviewers for careful reading and helpful comments. The work of Zhucui Jing and Ming Li are partially supported by the research grant 11101401 from NSFC for Youth. 


\section{References}

[1] D.P. Bertsekas. Nonlinear Programming. Athena Scientific, Belmont, 1999.

[2] J.F. Cai, R. Chan and Z. Shen. A framelet-based image inpainting algorithm. Appl. Comput. Harmon. Anal., 24(2):131-149, 2008. http://dx.doi.org/10.1016/j.acha.2007.10.002.

[3] J.F. Cai, H. Ji, C. Liu and Z. Shen. Blind motion deblurring from a single image using sparse approximation. In Computer Vision and Pattern Recognition, 2009, pp. 104-111, 2009.

[4] J.F. Cai, S. Osher and Z. Shen. Split bregman methods and frame based image restoration. Multiscale Model. Simul. SIAM Interdiscip. J., 8(2):337-369, 2009. http://dx.doi.org/10.1137/090753504.

[5] R.A. Crowther. Procedures for three-dimensional reconstruction of spherical viruses by fourier synthesis from electron micrographs. Philos. Trans. R. Soc. Lond. Ser. B Biol. Sci., 261(837):221-230, 1971. http://dx.doi.org/10.1098/rstb.1971.0054.

[6] R.A. Crowther, D.J. DeRosier and A. Klug. The reconstruction of a threedimensional structure from projections and its application to electron microscopy. Proc. Roy. Soc., Ser. A, 317(1530):319-340, 1970. http://dx.doi.org/10.1098/rspa.1970.0119.

[7] I. Daubechies, M. Defrise and C. DeMol. An iterative thresholding algorithm for linear inverse problems with a sparsity constraint. Comm. Pure Appl. Math, 57(11):1413-1457, 2004. http://dx.doi.org/10.1002/cpa.20042.

[8] D.J. DeRosier and A. Klug. Reconstruction of three dimensional structures from electron micrographs. Nature, 217(5124):130-134, 1968. http://dx.doi.org/10.1038/217130a0.

[9] B. Dong, A. Chien, Z. Shen and S. Osher. A new multiscale representation for shapes and its application to blood vessel recovery. SIAM J. Sci. Comput., 32(4):1724-1739, 2010. http://dx.doi.org/10.1137/09076043X.

[10] B. Dong, H. Ji, J. Li, Z. Shen and Y. Xu. Wavelet frame based blind image inpainting. Appl. Comput. Harmon. Anal., 32(2):268-279, 2012. http://dx.doi.org/10.1016/j.acha.2011.06.001.

[11] B. Dong, J. Li and Z. Shen. X-ray CT image reconstruction via wavelet frame based regularization and radon domain inpainting. J. Sci. Comput., 54(2-3):333349, 2013. http://dx.doi.org/10.1007/s10915-012-9579-6.

[12] J. Frank. Electron Tomography Methods for Three-Dimensional Visualization of Structures in the Cell. Springer, New York, second edition, 2006.

[13] J. Frank. Three-dimensional electron microscopy of macromolecular assemblies. Oxford University Press, New York, 2006.

[14] S.D. Fuller, S.J Butcher, R.H. Cheng and T.S. Baker. Three-dimensional reconstruction of icosahedral particles - the uncommon line. J. Struct. Biol., 116(1):48-55, 1996. http://dx.doi.org/10.1006/jsbi.1996.0009.

[15] N. Grigorieff. Frealign: High-resolution refinement of single particle structures. J. Struct. Biol., 157(1):117-125, 2007. http://dx.doi.org/10.1016/j.jsb.2006.05.004.

[16] M.V. Heel. Angular reconstruction: a posteriori assignment of projection directions for 3D reconstruction. Ultramicroscopy, 21(2):111-124, 1987. http://dx.doi.org/10.1016/0304-3991(87)90078-7. 
[17] M.V. Heel, B. Gowen, R. Matadeen, E.V. Orlova, R. Finn, T. Pape, D. Cohen, H. Stark, R. Schmidt, M. Schatz and A. Patwardhan. Single-particle electron cryo-microscopy: towards atomic resolution. Q. Rev. Biophys., 33(4):307-369, 2000. http://dx.doi.org/10.1017/S0033583500003644.

[18] W.C. Lau and J.L. Rubinstein. Subnanometre-resolution structure of the intact Thermus thermophilus H+-driven ATP synthase. nature, 481(7380):214-219, 2012 .

[19] M. Li, Z. Fan, H. Ji and Z. Shen. Wavelet frame based algorithm for 3d reconstruction in electron microscopy. SIAM J. Sci. Comput., 36(1):B45-B69, 2014. http://dx.doi.org/10.1137/130914474.

[20] M. Li, G. Xu, C. O.S. Sorzano, F. Sun and C. L. Bajaj. Single-particle reconstruction using $L^{2}$-gradient flow. J. Struct. Biol., 176:259-267, 2011. http://dx.doi.org/10.1016/j.jsb.2011.08.005.

[21] S.J. Ludtke, P.R. Baldwin and W. Chiu. EMAN: semiautomated software for high-resolution single-particle reconstructions. J. Struct. Biol., 128(1):82-97, 1999. http://dx.doi.org/10.1006/jsbi.1999.4174.

[22] J.A. Nelder and R. Mead. A simplex method for function minimization. Comput. J., 7:308-313, 1965. http://dx.doi.org/10.1093/comjnl/7.4.308.

[23] P.A. Penczek, R.A. Grasucci and J. Frank. The ribosome at improved resolution: New techniques for merging and orientation refinement in 3D cryo-electron microscopy of biological particles. Ultramicroscopy, 53(3):251-270, 1994.

[24] H.R. Saibil. Macromolecular structure determination by cryo-electron microscopy. Acta Cryst. D, 56(1):1215-1222, 2000. http://dx.doi.org/10.1107/S0907444900010787.

[25] C.O.S. Sorzano, R. Marabini, J. Velázquez-Muriel, J.R. Bilbao-Castro, S.H.W. Scheres, J.M. Carazo and A. Pascual-Montano. Xmipp: a new generation of an open-source image processing package for electron microscopy. J. Struct. Biol., 148(2):194-204, 2004. http://dx.doi.org/10.1016/j.jsb.2004.06.006. 